\title{
108. Intraoperative Lokalisation von Steinen in den Gallenwegen mit dem Festkörpersuchgerät
}

\author{
H.-D. Reiß
}

Allgemein-chirurgische Klinik der Medizinischen Hochschule Hannover im Krankenhaus Oststadt

\section{Intraoperative Localization of Stones in the Bile Ducts with an Audio Amplifier for Detection of Foreign Bodies}

Summary. For 3 years the firm of Siemens (Erlangen, W. Germany) has been selling an audio amplifier for the localization of calculi and pieces of glass and metal in wounds during surgery. When the apparatus comes into contact with any foreign bodies it transforms mechanical vibrations into electric impulses that are converted in turn to audible acoustic signals. The apparatus is very useful in revision of the common bile duct, but monitoring by X-ray or cholangioscope is still essential.

Key words: Calculi, localization of - Foreign bodies, detection with audio amplifier.

Zusammenfassung. Seit 3 Jahren befindet sich ein Festkörpersuchgerät (Fa. Siemens, Erlangen) zur intraoperativen Steinlokalisation sowie zur Lokalisation von Glassplittern und Metallteilen in Wunden im Handel. Das Gerät wandelt die bei der Berührung von Konkrementen, etc. mit Sonden entstehenden mechanischen Schwingungen in elektrische Spannungsimpulse um und setzt diese in hörbare akustische Signale um. Bei Choledochusrevisionen ist das Gerät empfehlenswert, ein Verzicht auf die intraoperative Röntgenkontrolle oder zusätzliche Choledochoskopie ist dadurch nicht möglich.

Schlïsselwörter: Steinlokalisation - Festkörpersuchgerät.

\section{Transjuguläre Leberdiagnostik: Cholangiographie, Biopsie, Portographie}

\author{
M. Thelen, K. J. Paquet, D. Schulz und P. Thurn \\ Radiologische Univ. Klinik, Röntgen-Abt. der Chirurgie Venusberg, D-5300 Bonn 1
}

\section{Diagnosis of Liver Conditions by the Transjugular Approach: Cholangiography, Biopsy, Portography}

Summary. The transjugular approach to the liver allows cholangiography, liver biopsy and retrograde portography. For cholangiography it is a prerequisite that the intrahepatic biliary tract be dilated. Biopsy from the right or left lobe allows differentiation between intra- and extrahepatic cholestasis. Retrograde portography is indicated for primary tumors of the liver and tumoral or metastatic extension to the liver. In portal hypertension portal hemodynamics can be determined before and after shunting operations.

Key words: Cholangiography, transjugular - Portography, transjugular.

Zusammenfassung. Der transjugulare Leberzugang bietet durch die Cholangiographie, Leberbiopsie und retrograde Portographie Erweiterungsmöglichkeiten der Leberdiagnose. Voraussetzung für die Cholangiographie sind gestaute intrahepatische Gallenwege. Die Leberbiopsie aus dem re. oder li. Leberlappen ermöglicht die Differenzierung zwischen intra- und extrahepatischer Cholostase. Die retrograde Portographie ist indiziert bei: Lebertumoren, Verdacht auf Tumoreinbruch in die Leber. Bei portaler Hypertension erlaubt sie vor und nach Shuntoperationen Aussagen über die Pfortaderhämodynamik.

Schliisselwörter: Transjuguläre Cholangiographie - Portographie. 